\title{
A serial study of platelet reactivity throughout the first six months after myocardial infarction: its modification by sulphinpyrazone
}

\author{
M.M. Kubik and S.G.N. Richardson
}

Departments of Medicine and Haematology, Burton Road Hospital, Dudley, West Midlands DYI 3BX, UK.

\begin{abstract}
Summary: Platelet reactivity was studied immediately after and throughout the first 6 months following myocardial infarction. Its modification by sulphinpyrazone was observed.

Out of 65 consecutive patients admitted to the coronary care unit, ten did not meet the protocol criteria. Fifty five received either placebo or sulphinpyrazone in a double-blind trial for 6 months, treatment being started within 6 days of infarction. Forty four patients completed the study (19 on placebo and 25 on sulphinpyrazone). An additional ten patients commenced treatment within one day of infarction and were studied daily for one week. Platelet hyper-reactivity was demonstrable in all patients at presentation and persisted throughout the 6 months in the control group. This effect was reversed by sulphinpyrazone, the modification of reactivity being established by the third day of treatment.
\end{abstract}

\section{Introduction}

Platelet hyper-reactivity is observed in patients after myocardial infarction ${ }^{1}$ and may still be detectable 3 months to 2 years later. ${ }^{2,3}$ It is likely that platelets play an important part in the pathogenesis of infarction because of their atherogenic properties, by occluding vessels with circulating platelet aggregates or by producing vasoconstricting agents locally.

Two- major trials showed that sulphinpyrazone reduced the incidence of sudden death ${ }^{4}$ and reinfarction $^{5}$ in the first 6 months after myocardial infarction. The presentation and interpretation of data in the first of these studies were criticized, but an independent reevaluation of the original material confirmed a favourable trend towards overall survival of patients treated with sulphinpyrazone. ${ }^{6}$ The mode of action of the drug in this context is unclear. The suggestion that it has anti-arrhythmic properties was not confirmed by Wilcox et al. ${ }^{7}$ nor by Kubik (unpublished observations). An anti-platelet action is more likely. Wallis ${ }^{8}$ has shown that sulphinpyrazone inhibits the enzyme cyclo-oxygenase and prostaglandin synthesis. When given to normal subjects, the drug impairs platelet aggregation induced by arachidonic acid (a prostaglandin precursor) and reduces the synthesis of malondialdehyde (an end product of platelet prostaglandin metabolism). ${ }^{9}$

For these reasons, arachidonic acid-induced platelet

Correspondence: M.M. Kubik, M.D., F.R.C.P.

Accepted: 21 October 1986 aggregation and malondialdehyde production were measured serially in the immediate post-infarction period and throughout the first 6 months after myocardial infarction. A double-blind trial of sulphinpyrazone was employed to see if the drug modified platelet behaviour during this period.

\section{Patients and methods}

All patients with a presumptive diagnosis of myocardial infarction admitted to the coronary care unit were evaluated for inclusion in the study. Only those in whom peptic ulcer had been diagnosed within the previous 3 years, those with a known hypersensitivity to pyrazole derivatives, those taking anti-platelet or anti-coagulant therapy and those on non-steroidal anti-inflammatory agents within 2 weeks of admission were not considered.

Sixty five consecutive patients were accepted in the study. Ten were withdrawn after baseline investigations because they did not meet the inclusion criteria. In three, serial electrocardiograms and cardiac enzyme levels did not confirm myocardial infarction, in five the serum creatinine was raised and in two the serum uric acid was above $0.5 \mathrm{mmol} / 1$. The 55 remaining patients were randomized in double-blind fashion to receive sulphinpyrazone or placebo for 6 months.

In an attempt to prevent or minimize the rise in urea and creatinine reported by Wilcock et al. ${ }^{7}$ in patients 
given sulphinpyrazone shortly after myocardial infarction, the drug was started in low dose $(200 \mathrm{mg})$ for 4 days, increased to $200 \mathrm{mg}$ b.d. for a further 3 days and then to $400 \mathrm{mg}$ b.d. for the remainder of the 6 month study period. Treatment commenced within 2 to 6 days of infarction to fit in with laboratory routine. Patients were evaluated at days 3 to 4,7 to 8 and between days 10 and 14 after starting treatment, and thereafter at day 28 and monthly for 6 months. They were examined again one month after the therapy was discontinued.

To study the effects of treatment immediately after infarction, ten additional patients, selected by the same criteria as those in the main group, started sulphinpyrazone or placebo within 24 hours of infarction and were tested daily for 7 days.

Normal ranges of arachidonic acid-induced platelet aggregation and malondialdehyde production were established from a group of 50 healthy subjects.

\section{Method}

Blood samples were taken from an arm vein, with minimum stasis, one to two hours after a dose of sulphinpyrazone had been given. Haemoglobin red cell and white cell counts were performed on a Coulter $S$ and platelet counts on a Coulter 'Thrombocounter'. Erythrocyte sedimentation rate was measured using the Westergren technique. Serum urea and urate were estimated using a Vickers SP 120 multi-channel analyser and lactate dehydrogenase and aspartate transaminase using a Baker centrifugal analyser. Blood for platelet function tests was taken into 0.1 vol $0.1 \mathrm{~N}$ sodium citrate. Platelet rich plasma was prepared by centrifugation of blood samples at $150 \mathrm{~g}$ for 10 minutes and platelet poor plasma at $1500 \mathrm{~g}$ for 10 minutes. The platelet count of platelet rich plasma was adjusted to $300-50 \times 10^{9} / 1$ by addition of platelet poor plasma.

Arachidonic acid aggregation The method described by Maguire et al. ${ }^{10}$ was used. Platelet aggregation induced by different concentrations of sodium arachidonate was measured on each platelet rich plasma sample using a Coulter 440 dual channel aggregometer. The time take for a $10 \%$ increase in light transmission was plotted against the sodium arachidonate concentration and the amount of sodium arachidonate required to cause a $10 \%$ reduction in one minute calculated. This was taken as an index of platelet responsiveness.

Malondialdehyde synthesis Modifications were made $\bar{c}$ to the method of Maguire et al..$^{10}$ Platelets were induced to aggregate by the addition of $50 \mu \mathrm{g}$ adenosine diphosphate (Sigma UK) to $3 \mathrm{ml}$ of platelet rich $\mathbb{\mathbb { D }}$ plasma. When large aggregates had formed, $3 \mathrm{ml}$ ethylene diamine tetracetic acid buffer ( $\mathrm{pH}$ 6.5) was added and the suspension centrifuged at $250 \mathrm{~g}$ for 5 minutes at room temperature. The supernatant was discarded and the platelet button resuspended in $2.5 \mathrm{ml}$ of dextrose-saline buffer (pH 6.5). A platelet $\overparen{8}$ count was performed on this sample. Malondialde- 3 hyde synthesis was induced by the addition of $10 \mu 1$ के sodium arachidonate (concentration $6.6 \mathrm{mmol}$ ) with incubation at $37^{\circ} \mathrm{C}$ for 15 minutes and was estimated using the thiobarbituric acid method. ${ }^{12,13}$ The result $\omega$ was corrected for the sample platelet count and expressed in nmol malondialdehyde $/ 10^{9}$ platelets. Sul- 음 phinpyrazone levels were measured in heparinized plasma using the method described by Bradbrook et al. ${ }^{14}$ The biochemical, haematological and platelet function data were analysed using the Mann-Whitney $\overrightarrow{0}$ test.

\section{Results}

Of the 55 patients admitted to the study, 27 received sulphinpyrazone and 28 placebo. The age and sex distribution of the two groups was comparable. The incidence of previous infarction, angina, hypertension, diabetes and smoking habits was similar as was treatment with beta-blockers, diuretics and calcium antagonists (Table I). During the course of the study, four patients died suddenly, two in hospital (cardiac arrest with asystole) and two at home (cause of death not established). Three of these four patients were receiving placebo and one sulphinpyrazone. One patient was referred for coronary artery bypass, another had a pulmonary embolus and was anticoagulated. One patient inadvertently took a course of aspirin and four were lost to follow up. Data on the 44 patients completing the study (25 on $\tilde{N}$

Table I Age and sex distribution, and incidence of previous infarction, angina and hypertension in 55 patients entering the study

\begin{tabular}{|c|c|c|c|c|c|c|c|}
\hline \multirow[b]{2}{*}{ No. of patients treated } & \multicolumn{2}{|c|}{ Age (years) } & \multicolumn{2}{|c|}{ Sex } & \multirow{2}{*}{$\begin{array}{c}\text { Previous } \\
\text { infarct }\end{array}$} & \multirow{2}{*}{$\begin{array}{l}\text { Previous } \\
\text { angina }\end{array}$} & \multirow[b]{2}{*}{ Hypertension } \\
\hline & mean & range & $M$ & $F$ & & & \\
\hline Sulphinpyrazone (27) & 57.8 & $42-74$ & 26 & 1 & 4 & 5 & 10 \\
\hline Placebo (28) & 55.7 & $42-73$ & 25 & 3 & 8 & 12 & 6 \\
\hline
\end{tabular}




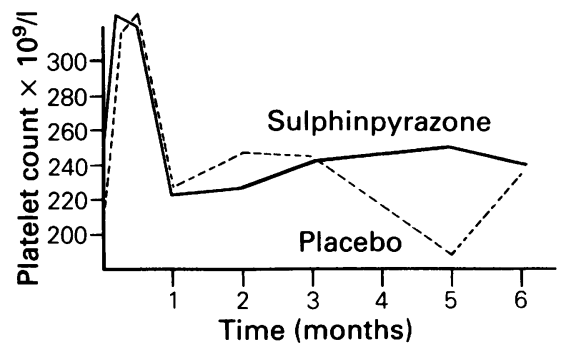

Figure 1 Mean platelet counts $\left(\times 10^{9} / 1\right)$. (Differences not significant.)

sulphinpyrazone and 19 on placebo) were analysed. All patients showed an initial rise in platelet count, maximal at days 7-14. The tendency for counts to be higher in the sulphinpyrazone group from the fourth to the sixth month was not statistically significant (Figure 1).

Pre-treatment platelet function tests revealed an increased reactivity to arachidonic acid induced aggregation, in the patient group, compared with normal subjects $(P<0.01)$. This platelet hyper-responsiveness persisted in the placebo group throughout the study period, but did not occur in the sulphinpyrazone group (Figure 2). One month after treatment ceased, patients who had been on sulphinpyrazone showed the same high levels of platelet reactivity as those on placebo. Data from the ten patients in the early treatment group revealed that while platelet hyper-responsiveness was present within 24 hours of infarction, it became more marked over the first 3 days. The early trend was prevented by sulphin-

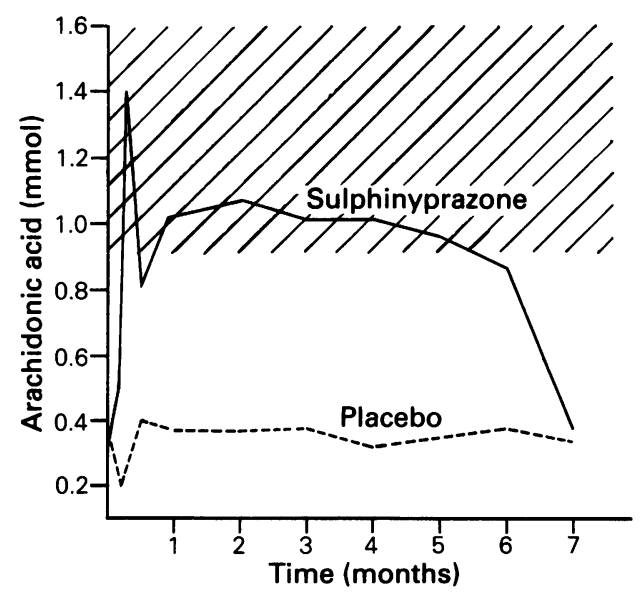

Figure 2 Concentration of arachidonic acid required to induced $10 \%$ aggregation in one minute: mean levels in sulphinpyrazone and placebo treated patients $(P<0.01$ at months 1, 2, 3 and 6); normal range.

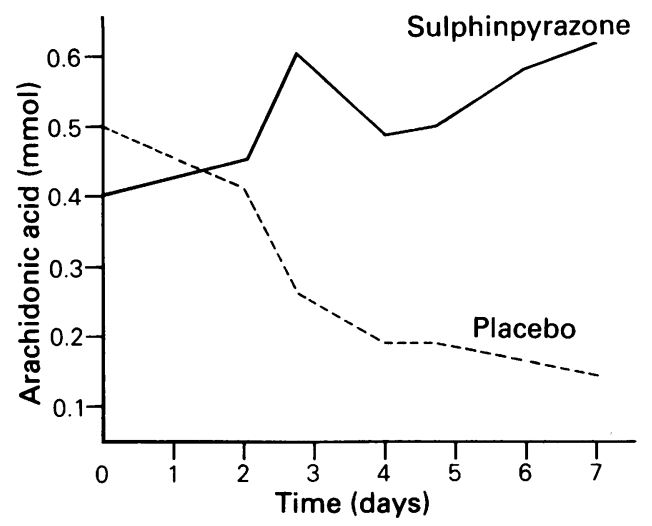

Figure 3 Arachidonic acid induced aggregation response during the first week after myocardial infarction.

pyrazone (Figure 3). In both treatment groups, malondialdehyde levels were lower than normal control values at entry into the study and during the first 2 months of treatment. While the mean levels in the sulphinpyrazone group were consistently lower throughout the treatment period, the differences were not statistically significant.

Haemoglobin and red cell values rose steadily in the placebo group during the period of study, but remained stable in the sulphinpyrazone patients. Though the levels were within the normal range, the difference between the two groups became significant at the third month and remained so thereafter without any biochemical evidence of haemolysis, or clinical suspicion of blood loss in the sulphinpyrazone treated patients (Figures 4, 5). White cell counts were similar in the two groups.

Serum urea and creatinine were higher during the first 7 days of treatment in the sulphinpyrazone treated group, though the differences were not statistically significant. Similar changes were also seen in the smaller study group, being apparent on the third day. The uricosuric effect of sulphinpyrazone was reflected by a significant fall in serum urate which was evident by the third day of therapy and which persisted throughout the treatment period. This fall provided a retrospective index of good patient compliance confirmed by serum drug assay levels. No patient had to be withdrawn because of an unacceptable deterioration in renal function and no patients complained of any symptoms attributable to sulphinpyrazone.*

\section{Discussion}

Enhanced platelet aggregation to adenosine diphosphate and collagen and increased platelet adhesiveness

* The authors will let any interested reader have a copy of the data. 


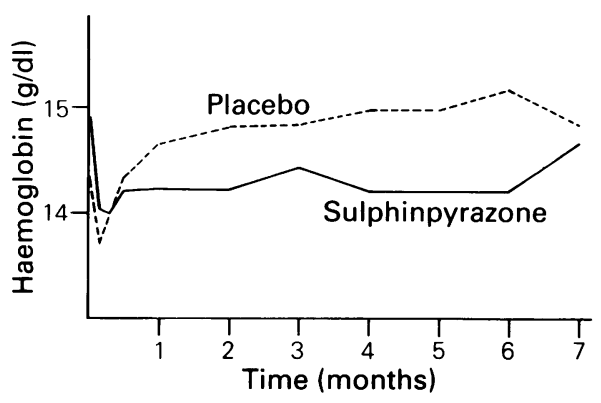

Figure 4 Mean haemoglobin levels in sulphinpyrazone and placebo treated patients $(P<0.05$ at month 2 ; $P<0.01$ at month 6).

have been demonstrated in patients with coronary and cerebral arterial insufficiency. ${ }^{14,15,16}$ Spontaneous platelet aggregration in vitro and an increase in circulating platelet aggregrates in vivo have been shown in coronary disease and after myocardial infarction. ${ }^{\prime}$ Activated platelets produce the potent vaso-constrictor and platelet aggregating agent thromboxane $A_{2}^{17,18}$ by oxidation of endogenous arachidonic acid. Sulphinpyrazone, a cyclo-oxygenase inhibitor, interferes with its synthesis. ${ }^{8}$ This drug, given to normal subjects, impairs arachidonic acidinduced platelet aggregation and reduces platelet malondialdehyde production. ${ }^{9}$ Szczeklik et al. ${ }^{2}$ found increased sensitivity to arachidonic acid-induced aggregation and more rapid platelet synthesis of thromboxane $\mathrm{A}_{2}$ in patients who had had a myocardial infarction at least 3 months previously. Agents like sulphinpyrazone which inhibit cyclo-oxygenase, reduce the sensitivity of platelets to arachidonic acidinduced aggregation. ${ }^{8}$

In our study, patients after myocardial infarction showed a significantly increased sensitivity to arachidonic acid throughout the seven months of observation, the changes being already apparent when the patients were first evaluated within 24 hours of infarction, but becoming more marked over the next 3 days. Similar increased platelet reactivity to other aggregating agents like adenosine diphosphate and adrenaline has been reported in myocardial infarction up to 2 years after the attack ${ }^{3,16}$ though Yamazaki et $a l .{ }^{19}$ found it limited to the acute phase of coronary occlusion. Szczeklik et al. ${ }^{2}$ demonstrated increased responsiveness to arachidonic acid persisting beyond 3 years after myocardial infarction. In our study, untreated patients showed persistent hyper-responsiveness throughout the 7 months of observation.

Sulphinpyrazone treatment reversed this hyperreactivity to levels near to or within the normal range. The effect became apparent on the third day of treatment and persisted throughout the 6 months of

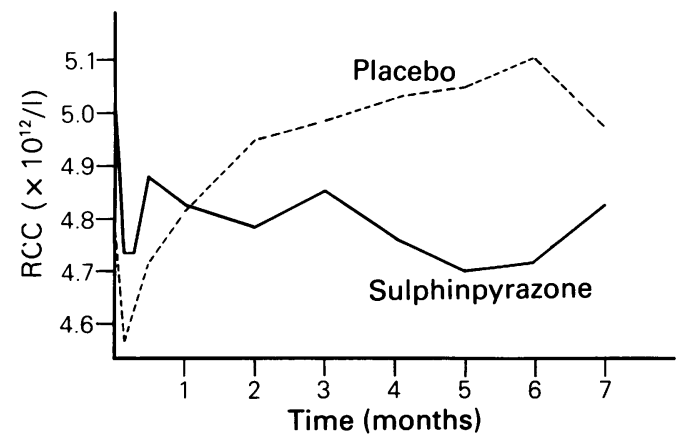

Figure 5 Mean red cell count (RCC) levels in sulphinpyrazone and placebo treated patients $(P<0.01$ at months 2, 3 and 6).

therapy. Re-evaluation one month after the drug was stopped showed a return to the same high level of 을 reactivity as in the untreated patients. Maguire et al. ${ }^{9} \rightarrow$ demonstrated that sulphinpyrazone reduced the $\bar{c}$ production of malondialdehyde in normal subjects. Such reduction was not apparent in our study of $\overrightarrow{0}$ patients after myocardial infarction nor was 욕 observed by Latour et al. $^{3}$ in patients taking sulphi pyrazone 2 months after infarction.

If malondialdehyde levels, like thromboxane $\mathrm{A}_{2}{ }^{2}$ are an indicator of the magnitude of prostaglandin synthesis, high levels would be expected in the post-infarction period. In our study, malondialdehyde production was below normal in treated and untreated patients in the first 2 months after infarction. This may reflect consumption of activated platelets, leaving a population of older less reactive ones, which Frampton et $a l^{20}$ showed to remain hyper-responsive to arachidonic acid. Alternatively, as Smith et al. ${ }^{12}$ point out, a decrease in malondialdehyde production may not necessarily result from a decrease in prostaglandin endoperoxide synthesis but be due to increased production of other stable end-products and $\mathrm{PGF}_{2 \alpha}$.

The steady and significant rise in haemoglobin and red cell count that occurred in the placebo but not in the treatment group remains unexplained. There was no evidence of bone marrow suppression or $\mathcal{N}$ haemolysis and no clinical suspicion of blood loss. N While the haemocrit is a major determinant of whole blood viscosity and hence of blood-flow, it is unlikely $\omega$ that the small difference seen here would modify flow properties and contribute to the beneficial clinical effects of sulphinpyrazone. Our study demonstrates a reversal of platelet hyper-reactivity after a few days' treatment with sulphinpyrazone. This effect was maintained throughout 6 months of treatment and was lost when therapy was withdrawn. The potential in vivo 
benefits of this modification of platelet behaviour in vitro are supported by the observations of Cortellaro et al. ${ }^{21}$ who showed correction of the shortened platelet production time (and hence presumably of the platelet survival time) in a small group of patients, treated with sulphinpyrazone, who had been entered in the Anturan Reinfarction Italian Study. These patients, and those studied by Latour et al. ${ }^{3}$ started sulphinpyrazone some months after infarction. Our patients began treatment within 6 days of infarct. The reversal of platelet hyper-reactivity was rapid even in those starting the drug in the first 24 hours. Since most complications occur in the immediate post-infarction period, early therapy might be of particular benefit. The commencement of treatment in step-wise fashion avoided the significant changes in renal function which had been observed by Wilcock et al. ${ }^{7}$ using higher initial doses. A similar incremental dose schedule was regarded as safe in cardiovascular azotemic patients by Palummeri et al. ${ }^{22}$ The modification of platelet function was seen by the third day of treatment when the patients were only receiving quarter dose $(200 \mathrm{mg}$ daily). This poses the question whether lower doses

\section{References}

1. Wu, K.K. \& Hoak, J.C. Spontaneous platelet aggregation in arterial insufficiency; mechanisms and implications. Thromb Haemost (Stuttg) 1976, 35: 702-711.

2. Szczeklik, A., Gryclewski, R.J., Musial, J., Grodzinska, L., Serwonska, M. \& Marcinkiewicz, E. Thromboxane generation and platelet aggregation in survivors of myocardial infarction. Thromb Haemost (Stuttg) 1978, 40: $66-74$.

3. Latour, J.G., Theroux, P. \& Bourassa, M.G. Sulphinpyrazone decreases epinephrine induced platelet aggregration after myocardial infarction. Am J Cardiol 1982, 50: 938-944.

4. The Anturan Reinfarction Trial Research Group. Sulfinpyrazone in the prevention of sudden death after myocardial infarction. $N$ Engl $J$ Med 1980, 302: 250256.

5. Report from Anturan Reinfarction Italian Study. Sulphinpyrazone in post-myocardial infarction. Lancet 1982, i: $237-242$.

6. The Anturan Reinfarction Trial: re-evaluation of outcome. Report of the Anturan Reinfarction Trial Policy Committee. $N$ Engl J Med 1982, 306: 1005-1008.

7. Wilcock, R.G., Richardson, D., Hampton, J.R., Mitchell, J.R.A. \& Banks, D.C. Sulphinpyrazone in acute myocardial infarction: studies on cardiac rhythm and renal function. $\mathrm{Br}$ Med $J$ 1980, 281: 531-534.

8. Wallis, R.B. Mechanism of action of sulphinpyrazone. Thromb Research 1983, Suppl IV: 31-38.

9. Maguire, E.D., Pay, G.F., Turney, J. et al. The effect of two different dosage regimens of sulphinpyrazone on platelet function ex vivo and blood chemistry in man. Haemostasis 1981, 10(3): 153-164.

10. Maguire, E.D., Pay, G.F., Wallis, R.B. \& White, A.M. used throughout would have been adequate. There is some evidence that calcium antagonists reduce platelet reactivity. ${ }^{23,24}$ Fortunately patients taking calcium antagonists were equally divided between the treatment and placebo group and no additional antiplatelet effect was observed in these patients. Although three of the four deaths occurring during the 7 month period of observation occurred in the placebo group, the number of patients studied is too small and the period of treatment too short to evaluate the effect of the drug on survival. The data from this study, in conjunction with other evidence, support the view that the beneficial effects of sulphinpyrazone after myocardial infarction are related to its anti-platelet properties. It may be that treatment with a lower dose and commenced within 24 hours of infarction would be more appropriate and equally effective.

\section{Acknowledgements}

We wish to thank Drs C.W.F. Clarke and N.H. Stentiford for allowing us to study patients under their care and Geigy Pharmaceuticals for logistical support.

Prolonged inhibition of ex vivo sodium arachidonateinduced platelet aggregation and malondialdehyde (MDA) production by sulphinpyrazone (Anturan) in man. Thromb Res 1981, 21: 321-327.

11. Stuart, M.J., Murphy, S. \& Oski, F.A. A simple nonradioisotope technique for the determination of platelet life-span. $N$ Engl J Med 1975, 292: 1310-1313.

12. Smith, J.B., Ingerman, C.M. \& Silver, M.J. Malondialdehyde formation as an indicator of prostaglandin production by human platelets. J Lab Clin Med 1976, 88: 167-173.

13. Bradbrook, I.D., John, V.A., Morrison, P.J., Rogers, H.J. \& Spector, R.G. Pharmacokinetics of single doses of sulphinpyrazone and its major metabolites in plasma and urine. Br J Clin Pharmacol 1982, 13: 177-185.

14. Dreyfus, F. \& Zahavi, J. Adenosine disphosphate induced platelet aggregation in myocardial infarction and ischaemic heart disease. Atherosclerosis 1973, 17: 107-120.

15. Hampton, J.R. \& Gorlin, R. Platelet studies in patients with coronary artery disease and in their relatives. $\mathrm{Br}$ Heart J 1972, 34: 465-471.

16. Salky, N. \& Dugdale, M. Platelet abnormalities in ischaemic heart disease. Am J Cardiol 1976, 32: 612-617.

17. Hamberg, M., Svenson, J. \& Samuelsson, B. Thromboxanes: a new group of biologically active compounds derived from prostaglandin endoperoxides. Proc Natl Acad Sci USA 1975, 72: 2994-2998.

18. Bunting, S., Moncada, S., Vane, J.R. The effect of prostaglandin endoperoxides and thromboxane $\mathrm{A}_{2}$ on strips of rabbit coeliac artery and certain other smooth muscle preparations. Br J Pharmacol 1976, 57: 462P-463P.

19. Yamazaki, H., Sano, T., Asano, T., Hidaka, H. Hyper- 
aggregability of platelets in thromboembolic disorders. Thromb Res 1976, 8 (Suppl II): 217-225.

20. Frampton, G. In vivo platelet aggregation and in vitro hyperaggregability to arachidonic acid in renal allograft recipients. Kidney Int 1983, 23: 506-513.

21. Cortellaro, M., Boschetti, C., Beggi, P. \& Polli, E.E. In vivo platelet hyperactivity and factor VIII related antigen increased long after myocardial infarction. Scand $J$ Haematol 1981, 26: 106-114.

22. Palummeri, E., Borghi, C., Di Ruvo, R.. Dacco. L. \&
Passeri, M. Sulphinpyrazine in cardiovascular elderly azotemic patients: a proposal of a 'guided' incremental dose schedule. J Int Med Res 1984, 12: 271-276.

23. Johnsson, $H$. Effects by nifedipine (Adalat $(R)$ ) on platelet function in vitro and in vivo. Thromb Res 1981, 21: 523-528.

24. Barnathan, E.S., Adonizio, V.P. \& Shattil, S.L. Interaction of verapamil with human platelet $\alpha$-adrenergic receptors. Am J Physiol 1982, 242: H19-H23. 